\title{
A note on stability of SPDEs driven by $\alpha$-stable noises
}

\author{
Jiying Wang* and Yulei Rao
}

"Correspondence: kellywang782@hotmail.com Business School, Central South University, Changsha, Hunan 410083, China

\begin{abstract}
In this paper, by the Minkovski inequality and the semigroup method we discuss the stability of mild solutions for a class of SPDEs driven by $\alpha$-stable noise, and the methods are also generalized to deal with the stability of SPDEs driven by subordinated cylindrical Brownian motion and fractional Brownian motion, respectively.
\end{abstract}

MSC: $60 \mathrm{H} 15 ; 60 \mathrm{G} 15$

Keywords: stochastic partial differential equation; $\alpha$-stable process; exponential stability; subordinated cylindrical Brownian motion; fractional Brownian motion

\section{Introduction}

The stability of stochastic partial differential equations (SPDEs) driven by Brownian motions or Lévy processes has been well established; see, e.g., Bao and Yuan [1], Bao et al. [2, 3], Chow [4], Liu [5] and Yuan and Bao [6], to name a few, where the noise processes are assumed to be square integrable. However, such restriction clearly rules out the interesting $\alpha$-stable processes since Wiener noise and Poisson-jump noise have arbitrary finite moments, while $\alpha$-stable noise only has finite $p$ th moment for $p \in(0, \alpha)$ with $\alpha<2$. Recently, stochastic equations driven by $\alpha$-stable processes, which have plenty of applications in physics due to the fact that the $\alpha$-stable noise exhibits the heavy tailed phenomenon, e.g., Solomon et al. [7], receives great attention. For example, Priola and Zabczyk [8] gave a proper starting point on the investigation of structural properties of SPDEs driven by an additive cylindrical stable noise, Dong et al. [9] studied the ergodicity of stochastic Burgers equations driven by $\alpha / 2$-subordinated cylindrical Brownian motion with $\alpha \in(1,2)$, and Zhang [10] established the Bismut-Elworthy-Li derivative formula for stochastic differential equations (SDEs) driven by $\alpha$-stable noise. For finite-dimensional cases, Wang [11] derived a gradient estimate for linear SDEs driven by $\alpha$-stable noise, and Wang [12] established the functional inequalities for Ornstein-Uhlenbeck processes driven by $\alpha$-stable noise by the sharp estimates of density function for rotationally invariant symmetric $\alpha$-stable Lévy processes. However, there are few papers on the asymptotic behavior of mild solution of SPDEs driven by $\alpha$-stable processes. In this note, we shall discuss the stability property of mild solutions of a class of SPDEs driven by $\alpha$-stable processes to close the gap. Due to the fact that $\alpha$-stable noise only has finite $p$ th moment for $p \in(0, \alpha)$ and that the stochastic evolution does not admit a stochastic differential, which leads to the Itô formula being unavailable, then some new tricks need to be put forward to overcome the difficulties brought about by $\alpha$-stable noise.

○2014 Wang and Rao; licensee Springer. This is an Open Access article distributed under the terms of the Creative Commons Attribution License (http://creativecommons.org/licenses/by/2.0), which permits unrestricted use, distribution, and reproduction in any medium, provided the original work is properly cited. 
This note is organized as follows: in Section 2 we apply the Minkovski inequality and the semigroup method to investigate the exponential stability of mild solutions for a class of SPDEs driven by $\alpha$-stable processes, and these tricks are extended to cope with the stability of mild solutions of SPDEs driven by subordinated cylindrical Brownian motion and fractional Brownian motion, respectively, in Section 3.

\section{Stability of SPDEs driven by $\alpha$-stable noise}

We firstly give an overview of stable processes. An $\mathbb{R}$-valued random variable $\eta$ is said to be stable with stability index $\alpha \in(0,2)$, scale parameter $\sigma \in(0, \infty)$, skewness parameter $\beta \in[-1,1]$, and location parameter $\mu \in(-\infty, \infty)$ if it has characteristic function of the form

$$
\phi_{\eta}(u)=\mathbb{E} \exp (i u \eta)=\exp \left\{-\sigma^{\alpha}|u|^{\alpha}(1-i \beta \operatorname{sgn}(u) \Phi)+i \mu u\right\}, \quad u \in \mathbb{R},
$$

where $\Phi=\tan (\pi \alpha / 2)$ for $\alpha \neq 1$ and $\Phi=-(2 / \pi) \log |u|$ for $\alpha=1$. We call $\eta$ is strictly $\alpha$ stable whenever $\mu=0$, and if, in addition, $\beta=0, \eta$ is said to be symmetric $\alpha$-stable. We refer to, e.g., Applebaum [13] and Sato [14], for more details of stable distributions. Let $\left(\mathbb{H},\langle\cdot, \cdot\rangle,\|\cdot\|_{\mathbb{H}}\right)$ be a real separable Hilbert space, and $Z(t)$ a cylindrical $\alpha$-stable process, defined by

$$
Z(t):=\sum_{k=1}^{\infty} \beta_{k}^{H} Z_{k}(t) e_{k}
$$

Here $\left\{e_{k}\right\}_{k \geq 1}$ is an orthonormal basis of $\mathbb{H},\left\{Z_{k}(t)\right\}_{k \geq 1}$ are independent, $\mathbb{R}$-valued, normalized, symmetric $\alpha$-stable Lévy processes defined on the stochastic basis $\left(\Omega, \mathcal{F},\left\{\mathcal{F}_{t}\right\}_{t \geq 0}, \mathbb{P}\right)$, and $\left\{\beta_{k}\right\}_{k \geq 1}$ is a sequence of positive numbers.

In this section, we shall consider an SPDE driven by $\alpha$-stable process in $\mathbb{H}$

$$
\mathrm{d} X(t)=\{A X(t)+b(t, X(t))\} \mathrm{d} t+\sigma(t) \mathrm{d} Z(t), \quad t>0,
$$

with initial value $X(0)=x$.

In this section we shall assume the following.

(A1) $(A, \mathcal{D}(A))$ is a self-adjoint compact operator on $\mathbb{H}$ such that $-A$ has discrete spectrum $0<\lambda_{1}<\lambda_{2}<\cdots<\lambda_{k}<\cdots, \lim _{k \rightarrow \infty} \lambda_{k}=\infty$ with corresponding eigenbasis $\left\{e_{k}\right\}_{k \geq 1}$ of $\mathbb{H}$. In this case, by [15, Theorem 6.26, p.185] and [15, Theorem 6.29, p.187], $A$ generates a $C_{0}$-semigroup $\mathrm{e}^{t A}, t \geq 0$, such that $\left\|\mathrm{e}^{t A}\right\| \leq \mathrm{e}^{-\lambda_{1} t}$, where $\|\cdot\|$ denotes the usual operator norm.

(A2) $b:[0, \infty) \times \mathbb{H} \rightarrow \mathbb{H}$ and there exist $L_{1}>0$ and $L_{2}>0$ such that

$$
\|b(t, x)-b(t, y)\|_{\mathbb{H}} \leq L_{1}\|x-y\|_{\mathbb{H}}, \quad t \geq 0, x, y \in \mathbb{H}
$$

and

$$
\|b(t, x)\|_{\mathbb{H}} \leq L_{2}\left(\gamma(t)+\|x\|_{\mathbb{H}}\right), \quad t \geq 0, x \in H
$$

where $\gamma:[0, \infty) \rightarrow[0, \infty)$ is a local integrable continuous function. 
(A3) $\sigma:[0, \infty) \rightarrow \mathbb{R}^{+}$is a continuous function such that

$$
\delta_{1}(t):=\sum_{k=1}^{\infty} \beta_{k}^{\alpha} \int_{0}^{t} \mathrm{e}^{-\alpha \lambda_{k}(t-s)} \sigma^{\alpha}(s) \mathrm{d} s, \quad t \geq 0,
$$

is locally bounded, i.e., $\delta_{1}(t)$ is bounded on the time interval $[0, T]$ for any $T>0$, where $\left\{\beta_{k}\right\}_{k \geq 1}$ is the sequence appearing in (2.1) and $\left\{\lambda_{k}\right\}_{k \geq 1}$ is the discrete spectrum of $A$.

Under (A1)-(A3), equation (2.2) has a unique mild solution, see, e.g., [8, Formula 5.3], that is, there exists a predictable $\mathbb{H}$-valued stochastic process $X(t)$ such that

$$
X(t)=\mathrm{e}^{t A} x+\int_{0}^{t} \mathrm{e}^{(t-s) A} b(t, X(s)) \mathrm{d} s+\int_{0}^{t} \mathrm{e}^{(t-s) A} \sigma(s) \mathrm{d} Z(s) .
$$

Next we recall the following Minkowski inequality, which plays a key role in revealing the stability property of SPDEs driven by $\alpha$-stable processes.

Lemma 2.1 Let $\left(\mathbb{S}_{1}, \mu_{1}\right)$ and $\left(\mathbb{S}_{2}, \mu_{2}\right)$ be two measurable spaces and $F: \mathbb{S}_{1} \times \mathbb{S}_{2} \rightarrow \mathbb{R}$ be measurable. Then, for any $q>1$,

$$
\left(\int_{\mathbb{S}_{2}}\left|\int_{\mathbb{S}_{1}} F(x, y) \mu_{1}(\mathrm{~d} x)\right|^{q} \mu_{2}(\mathrm{~d} y)\right)^{1 / q} \leq \int_{\mathbb{S}_{1}}\left(\int_{\mathbb{S}_{2}}|F(x, y)|^{q} \mu_{2}(\mathrm{~d} x)\right)^{1 / q} \mu_{1}(\mathrm{~d} y) .
$$

We now can state our main results in this section.

Theorem 2.2 Let (A1) and (A2) hold and assume further that

$$
\delta_{2}:=\sup _{t \geq 0}\left(\int_{0}^{t} \mathrm{e}^{\lambda_{1} s} \gamma(s) \mathrm{d} s\right)<\infty
$$

and

$$
\delta_{3}:=\sup _{t \geq 0}\left\{\mathrm{e}^{\alpha \lambda_{1} t} \sum_{k=1}^{\infty} \beta_{k}^{\alpha} \int_{0}^{t} \mathrm{e}^{-\alpha \lambda_{k}(t-s)} \sigma^{\alpha}(s) \mathrm{d} s\right\}<\infty .
$$

Then, for $\alpha \in(1,2), p \in(1, \alpha)$ and $L \in\left(0, \lambda_{1}\right)$, there exists $c_{1}>0$ such that

$$
\mathbb{E}\left(\|X(t)\|_{\mathbb{H}}^{p}\right) \leq c_{1} \mathrm{e}^{-p\left(\lambda_{1}-L\right) t} .
$$

That is, the solution is exponentially stable in the pth moment with the Lyapunov exponent $-\left(\lambda_{1}-L\right)$.

Proof Under (A1), (A2), and (2.5), (2.2) has a unique mild solution $X(t)$. Recall the fact that $\left(\mathbb{E}\|\cdot\|_{\mathbb{H}}^{p}\right)^{1 / p}, p \in(1, \alpha)$, is a norm, which will be utilized again and again. We then obtain from $(2.3)$

$$
\begin{aligned}
\left(\mathbb{E}\left(\|X(t)\|_{\mathbb{H}}^{p}\right)\right)^{1 / p} \leq & \left\|\mathrm{e}^{t A} x\right\|_{\mathbb{H}}+\left(\mathbb{E}\left(\left\|\int_{0}^{t} \mathrm{e}^{(t-s) A} b(s, X(s)) \mathrm{d} s\right\|_{\mathbb{H}}^{p}\right)\right)^{1 / p} \\
& +\left(\mathbb{E}\left(\left\|\int_{0}^{t} \mathrm{e}^{(t-s) A} \sigma(s) \mathrm{d} Z(s)\right\|_{\mathbb{H}}^{p}\right)\right)^{1 / p} \\
= & : I_{1}(t)+I_{2}(t)+\left(\mathbb{E}\left(\left\|I_{3}(t)\right\|_{\mathbb{H}}^{p}\right)\right)^{1 / p}
\end{aligned}
$$


By (A1), it is readily seen that

$$
I_{1}(t) \leq \mathrm{e}^{-\lambda_{1} t}\|x\|_{\mathbb{H}}
$$

Next, applying Lemma 2.1 and using (A1) and (A2) yield

$$
\begin{aligned}
I_{2}(t) & \leq \int_{0}^{t}\left(\mathbb{E}\left(\left\|\mathrm{e}^{(t-s) A} b(s, X(s))\right\|_{\mathbb{H}}^{p}\right)\right)^{1 / p} \mathrm{~d} s \\
& \leq \int_{0}^{t} \mathrm{e}^{-\lambda_{1}(t-s)}\left(\mathbb{E}\left(\|b(s, X(s))\|_{\mathbb{H}}^{p}\right)\right)^{1 / p} \mathrm{~d} s \\
& \leq L_{2} \int_{0}^{t} \mathrm{e}^{-\lambda_{1}(t-s)}\left(\mathbb{E}\left(\gamma(s)+\|X(s)\|_{\mathbb{H}}^{p}\right)\right)^{1 / p} \mathrm{~d} s \\
& \leq L_{2} \int_{0}^{t} \mathrm{e}^{-\lambda_{1}(t-s)}\left\{\gamma(s)+\left(\mathbb{E}\left(\|X(s)\|_{\mathbb{H}}^{p}\right)\right)^{1 / p}\right\} \mathrm{d} s,
\end{aligned}
$$

where we have also used the fact that $\left(\mathbb{E}\|\cdot\|_{\mathbb{H}}^{p}\right)^{1 / p}, p \in(1, \alpha)$, is a norm. By virtue of (2.1) and (A1), a direct calculation shows

$$
I_{3}(t)=\sum_{k=1}^{\infty} \Lambda_{k}(t) e_{k}
$$

where $\Lambda_{k}(t):=\int_{0}^{t} \beta_{k} e^{-\lambda_{k}(t-s)} \sigma(s) \mathrm{d} Z_{k}(s)$. Let $\left\{r_{k}\right\}_{k \geq 1}$ be a Rademacher sequence defined on a new probability space $\left(\Omega^{\prime}, \mathcal{F}^{\prime},\left\{\mathcal{F}_{t}^{\prime}\right\}_{t \geq 0}, \mathbb{P}^{\prime}\right)$, i.e., $r_{k}: \Omega^{\prime} \rightarrow\{1,-1\}$ are i.i.d. with $\mathbb{P}^{\prime}\left(r_{k}=1\right)=$ $\mathbb{P}^{\prime}\left(r_{k}=-1\right)=1 / 2$. Recall Khintchine's inequality: for a sequence of real numbers $\left\{c_{k}\right\}_{k \geq 1}$ and any $q>0$, there exists $c_{q}>0$ such that

$$
\left(\sum_{k \geq 1} c_{k}^{2}\right)^{1 / 2} \leq c_{q}\left(\mathbb{E}^{\prime}\left|\sum_{k \geq 1} r_{k} c_{k}\right|^{q}\right)^{1 / q} .
$$

Then, by (2.10) and following an argument similar to that of [8, Theorem 4.5], the stochastic evolution $I_{3}(t)$ has the estimation

$$
\begin{aligned}
\left(\mathbb{E}\left(\left\|I_{3}(t)\right\|_{\mathbb{H}}^{p}\right)\right)^{1 / p} & =\mathbb{E}\left(\sum_{k=1}^{\infty}\left(\int_{0}^{t} \beta_{k} \mathrm{e}^{-\lambda_{k}(t-s)} \sigma(s) \mathrm{d} Z_{k}(s)\right)^{2}\right)^{p / 2} \\
& \leq c_{p}^{p} \mathbb{E}^{\prime}\left|\sum_{k=1}^{\infty} r_{k} \Lambda_{k}(t)\right|^{p}=c_{p}^{p} \mathbb{E}^{\prime} \mathbb{E}\left|\sum_{k=1}^{\infty} r_{k} \Lambda_{k}(t)\right|^{p} \\
& \leq c_{p}\left(\sum_{k=1}^{\infty} \beta_{k}^{\alpha} \int_{0}^{t} \mathrm{e}^{-\alpha \lambda_{k}(t-s)} \sigma^{\alpha}(s) \mathrm{d} s\right)^{1 / \alpha}
\end{aligned}
$$

where $c_{p}>0$ is some constant. Substituting (2.8)-(2.11) into (2.7) gives

$$
\begin{aligned}
\mathrm{e}^{\lambda_{1} t}\left(\mathbb{E}\left(\|X(t)\|_{\mathbb{H}}^{p}\right)\right)^{1 / p} \leq & \|x\|_{\mathbb{H}}+L_{2} \int_{0}^{t} \mathrm{e}^{\lambda_{1} s}\left\{\gamma(s)+\left(\mathbb{E}\left(\|X(s)\|_{\mathbb{H}}^{p}\right)\right)^{1 / p}\right\} \mathrm{d} s \\
& +c_{p} \mathrm{e}^{\lambda_{1} t}\left(\sum_{k=1}^{\infty} \beta_{k}^{\alpha} \int_{0}^{t} \mathrm{e}^{-\alpha \lambda_{k}(t-s)} \sigma^{\alpha}(s) \mathrm{d} s\right)^{1 / \alpha} .
\end{aligned}
$$


Thus, by (2.4) and (2.5), we arrive at

$$
\mathrm{e}^{\lambda_{1} t}\left(\mathbb{E}\left(\|X(t)\|_{\mathbb{H}}^{p}\right)\right)^{1 / p} \leq\left\{\|x\|_{\mathbb{H}}+L_{2} \delta_{2}+c_{p} \delta_{3}^{1 / \alpha}\right\}+L_{2} \int_{0}^{t} \mathrm{e}^{\lambda_{1} s}\left(\mathbb{E}\left(\|X(s)\|_{\mathbb{H}}^{p}\right)\right)^{1 / p} \mathrm{~d} s
$$

In view of the Gronwall inequality,

$$
\mathrm{e}^{\lambda_{1} t}\left(\mathbb{E}\left(\|X(t)\|_{\mathbb{H}}^{p}\right)\right)^{1 / p} \leq\left\{\|x\|_{\mathbb{H}}+L_{2} \delta_{2}+c_{p} \delta_{3}^{1 / \alpha}\right\} \mathrm{e}^{L t}
$$

holds and the desired assertion (2.6) follows due to $L \in\left(0, \lambda_{1}\right)$.

In what follows, we establish an example to demonstrate that the condition (2.5) holds in many practical situations.

Example 2.3 Let $\sigma(t)=\mathrm{e}^{-\left(\lambda_{1}+\varepsilon\right) t}$, where $\varepsilon>0$ is a constant such that $\lambda_{1}+\varepsilon<\lambda_{2}$. A direct computation shows that

$$
\begin{aligned}
\mathrm{e}^{\alpha \lambda_{1} t} \sum_{k=1}^{\infty} \beta_{k}^{\alpha} \int_{0}^{t} \mathrm{e}^{-\alpha \lambda_{k}(t-s)} \sigma^{\alpha}(s) \mathrm{d} s & =\mathrm{e}^{\alpha \lambda_{1} t} \sum_{k=1}^{\infty} \beta_{k}^{\alpha} \mathrm{e}^{-\alpha \lambda_{k} t} \int_{0}^{t} \mathrm{e}^{\alpha\left(\lambda_{k}-\lambda_{1}-\varepsilon\right) s} \mathrm{~d} s \\
& =\beta_{1}^{\alpha} \int_{0}^{t} \mathrm{e}^{-\alpha \varepsilon s} \mathrm{~d} s+\mathrm{e}^{\alpha \lambda_{1} t} \sum_{k=2}^{\infty} \beta_{k}^{\alpha} \mathrm{e}^{-\alpha \lambda_{k} t} \int_{0}^{t} \mathrm{e}^{\alpha\left(\lambda_{k}-\lambda_{1}-\varepsilon\right) s} \mathrm{~d} s \\
& \leq \frac{\beta_{1}^{\alpha}}{\alpha \varepsilon}+\mathrm{e}^{-\alpha \varepsilon t} \sum_{k=2}^{\infty} \frac{\beta_{k}^{\alpha}}{\alpha\left(\lambda_{k}-\lambda_{1}-\varepsilon\right)} \\
& \leq \frac{\beta_{1}^{\alpha}}{\alpha \varepsilon}+\frac{\lambda_{2}}{\alpha\left(\lambda_{2}-\lambda_{1}-\varepsilon\right)} \sum_{k=2}^{\infty} \frac{\beta_{k}^{\alpha}}{\lambda_{k}}
\end{aligned}
$$

due to the increasing property of the spectrum $\left\{\lambda_{k}\right\}_{k \geq 1}$. If $\sum_{k=2}^{\infty} \frac{\beta_{k}^{\alpha}}{\lambda_{k}}<\infty$ holds, then both (A3) and (2.5) are satisfied. There are plenty of examples such that the condition $\sum_{k=2}^{\infty} \frac{\beta_{k}^{\alpha}}{\lambda_{k}}<$ $\infty$ holds, e.g., [8, Example 4.4]. Moreover, if $b(t, x)=c\left(\mathrm{e}^{-\delta t} \mathbf{1}+\bar{b}(x)\right), t \geq 0, x \in H, c>0$, $\delta \in\left(\lambda_{1}, \infty\right)$, where 1 is the identical operator on $H$ and $\bar{b}$ is Lipschitzian such that $\bar{b}(0)=0$, then (A2) and (2.4) holds.

Remark 2.1 To reveal the stability property of the mild solution of (2.1), we replace (A3) by a little bit strong condition (2.5), although (2.1) admits a unique mild solution in finitetime horizon under (A1)-(A3).

Remark 2.2 For $\alpha \in(0,1)$, if $b:[0, \infty) \times \mathbb{H} \rightarrow \mathbb{H}$ is bounded, then (2.1) has a unique mild solution under (A1)-(A3); see, e.g., [8, Proposition 5.1]. For such case, by the fundamental inequality

$$
(a+b+c)^{\theta} \leq a^{\theta}+b^{\theta}+c^{\theta}, \quad a, b, c>0, \theta \in(0,1),
$$

we have

$$
\mathbb{E}\left(\|X(t)\|_{\mathbb{H}}^{p}\right) \leq\left\|\mathrm{e}^{t A} x\right\|_{\mathbb{H}}^{p}+\mathbb{E}\left(\left\|\int_{0}^{t} \mathrm{e}^{(t-s) A} b(s, X(s)) \mathrm{d} s\right\|_{\mathbb{H}}^{p}\right)+\mathbb{E}\left(\left\|I_{3}(t)\right\|_{\mathbb{H}}^{p}\right),
$$


where $I_{3}(t)$ is defined as in (2.7). Then under appropriate conditions, we can also discuss the stability property of the mild solution of (2.2).

Remark 2.3 Due to the fact that stochastic evolution does not admit stochastic differential, we apply the semigroup method, not the Itô formula, to study the stability property of the mild solution of (2.2). Comparing with the current technique, we generally adopt the following procedure to discuss the exponential stability of (2.2): For $p \in(1, \alpha)$, by the inequality $(a+b+c)^{p} \leq 3^{p-1}\left(a^{p}+b^{p}+c^{p}\right), a, b, c>0$, (A1), and the Hölder inequality

$$
\begin{aligned}
\mathbb{E}\left(\|X(t)\|_{\mathbb{H}}^{p}\right) \leq & 3^{p-1}\left\{\mathrm{e}^{-p \lambda_{1} t}\|x\|_{\mathbb{H}}^{p}+\mathbb{E}\left(\int_{0}^{t} \mathrm{e}^{-\lambda_{1}(t-s)}\|b(s, X(s))\|_{\mathbb{H}} \mathrm{d} s\right)^{p}+\mathbb{E}\left(\left\|I_{3}(t)\right\|_{\mathbb{H}}^{p}\right)\right\} \\
= & 3^{p-1}\left\{\mathrm{e}^{-p \lambda_{1} t}\|x\|_{\mathbb{H}}^{p}+\mathbb{E}\left(\int_{0}^{t} \mathrm{e}^{-(p-1) / p \lambda_{1}(t-s)} \mathrm{e}^{-\left(\lambda_{1} / p\right)(t-s)}\|b(s, X(s))\|_{\mathbb{H}} \mathrm{d} s\right)^{p}\right. \\
& \left.+\mathbb{E}\left(\left\|I_{3}(t)\right\|_{\mathbb{H}}^{p}\right)\right\} \\
\leq & 3^{p-1}\left\{\mathrm{e}^{-\lambda_{1} t}\|x\|_{\mathbb{H}}^{p}+\lambda_{1}^{1-p} \mathbb{E} \int_{0}^{t} \mathrm{e}^{-\lambda_{1}(t-s)}\|b(s, X(s))\|_{\mathbb{H}}^{p} \mathrm{~d} s+\mathbb{E}\left(\left\|I_{3}(t)\right\|_{\mathbb{H}}^{p}\right)\right\} .
\end{aligned}
$$

Hence it follows that

$$
\mathrm{e}^{\lambda_{1} t} \mathbb{E}\left(\|X(t)\|_{\mathbb{H}}^{p}\right) \leq 3^{p-1}\left\{\|x\|_{\mathbb{H}}^{p}+\lambda_{1}^{1-p} \mathbb{E} \int_{0}^{t} \mathrm{e}^{\lambda_{1} s}\|b(s, X(s))\|_{\mathbb{H}}^{p} \mathrm{~d} s+\mathrm{e}^{\lambda_{1} t} \mathbb{E}\left(\left\|I_{3}(t)\right\|_{\mathbb{H}}^{p}\right)\right\} .
$$

Thus, carrying out a similar argument to that of Theorem 2.2, we conclude by the previous method that the Lyapunov exponent is dependent on $3^{p-1}, \lambda_{1}^{1-p}$ and $L_{2}$. However, by the technique introduced in the argument of Theorem 2.2, we find that the Lyapunov exponent is $-\left(\lambda_{1}-L_{2}\right)$, which only is dependent on $\lambda_{1}$ and $L_{2}$.

\section{Extension to SPDEs driven by subordinated cylindrical Brownian motions and fractional Brownian motions}

In the last section, we introduce some tricks to study the stability of mild solutions for a class of SPDEs driven by $\alpha$-stable noise. In this section, following these tricks, we proceed to investigate the stability of SPDEs driven by subordinated cylindrical Brownian motion and fractional Brownian motion, respectively. To begin with, we recall some notions. For $\alpha \in(0,2)$, let $S(t)$ be an $\alpha / 2$-stable subordinator defined on the probability space $\left(\Omega, \mathcal{F},\left\{\mathcal{F}_{t}\right\}_{t \geq 0}, \mathbb{P}\right)$, i.e., an increasing $\mathbb{R}$-valued Lévy process; see, e.g., Applebaum [13, pp.52-62] and Sato [14, Chapter 6], with Laplace transform

$$
\mathbb{E} \mathrm{e}^{-u S(t)}=\mathrm{e}^{-t|u|^{\alpha / 2}}, \quad u>0,
$$

see, e.g., [13, Example 1.3.14, p.51]. Let $\left\{W^{k}(t), t \geq 0\right\}_{k \in \mathbb{N}}$ be a sequence of independent standard one-dimensional Brownian motion defined on the probability space $\left(\Omega, \mathcal{F},\left\{\mathcal{F}_{t}\right\}_{t \geq 0}, \mathbb{P}\right)$. The subordinated cylindrical Brownian motion on $\mathbb{H}$ is defined by

$$
L(t):=\sum_{k=1}^{\infty} \beta_{k} W^{k}(S(t)) e_{k},
$$


where $S(t)$ is an $\alpha / 2$-stable subordinator independent of $\left\{W^{k}(t)\right\}_{k \geq 1},\left\{\beta_{k}\right\}_{k \geq 1}$ is a sequence of real numbers, and $\left\{e_{k}\right\}_{\geq 1}$ is an orthonormal basis of $\mathbb{H}$. Note that $Z^{k}(t):=W^{k}(S(t))$, which satisfies $Z^{k}(t)(\omega):=W^{k}(S(t)(\omega))(\omega)$ for each $\omega \in \Omega$, is a Lévy process by [13, Theorem 1.3.25, p.56] and an $\alpha$-stable process due to [13, Proposition 1.3.27, p.58].

In this section, we shall consider an SPDE driven by the subordinated cylindrical Brownian motion $L(t)$, defined by (3.1), on $\mathbb{H}$

$$
\mathrm{d} X(t)=\{A X(t)+b(t, X(t))\} \mathrm{d} t+\sigma(t) \mathrm{d} L(t), \quad t>0,
$$

with initial value $X_{0}=x$, where $\sigma:[0, \infty) \rightarrow \mathbb{R}$ is local integrable continuous function. Let (A1) and (A2) hold and assume further that

$$
\bar{\delta}(t):=\sum_{k=1}^{\infty} \beta_{k}^{2} \int_{0}^{t} \mathrm{e}^{-2 \lambda_{k}(t-s)} \sigma^{2}(s) \mathrm{d} s, \quad t \geq 0,
$$

is locally bounded. Then (3.2) admits a unique mild solution in a finite-time interval; see, e.g., [10, Proposition 4.3], that is, there exists a predictable $\mathbb{H}$-valued stochastic process $X(t)$ such that

$$
X(t)=\mathrm{e}^{t A} x+\int_{0}^{t} \mathrm{e}^{(t-s) A} b(s, X(s)) \mathrm{d} s+\int_{0}^{t} \mathrm{e}^{(t-s) A} \sigma(s) \mathrm{d} L(s) .
$$

One of our main results in this section is as follows.

Theorem 3.1 Let (A1), (A2), and (2.4) hold and assume further that

$$
\delta_{4}:=\sup _{t \geq 0}\left\{\mathrm{e}^{2 \lambda_{1} t} \sum_{k=1}^{\infty} \beta_{k}^{2} \int_{0}^{t} \mathrm{e}^{-2 \lambda_{k}(t-s)} \sigma^{2}(s) \mathrm{d} s\right\}<\infty .
$$

Then, for $\alpha \in(1,2), p \in(1, \alpha)$ and $L \in\left(0, \lambda_{1}\right)$, there exist $c_{2}>0$ and $\gamma \in\left(0, \lambda_{1}-L\right)$ such that

$$
\mathbb{E}\left(\|X(t)\|_{\mathbb{H}}^{p}\right) \leq c_{2} \mathrm{e}^{-p \gamma t} .
$$

In other words, the solution is exponentially stable in the pth moment, where the Lyapunov exponent is $-\gamma$.

Proof Also by the fact that $\left(\mathbb{E}\|\cdot\|_{\mathbb{H}}^{p}\right)^{1 / p}, p \in(1, \alpha)$, is norm, we deduce from (3.2) that

$$
\left(\mathbb{E}\left(\|X(t)\|_{\mathbb{H}}^{p}\right)\right)^{1 / p} \leq I_{1}(t)+I_{2}(t)+\left(\mathbb{E}\left(\left\|I_{4}(t)\right\|_{\mathbb{H}}^{p}\right)\right)^{1 / p},
$$

where $I_{1}(t)$ and $I_{2}(t)$ are defined as in (2.7), and $I_{4}(t)$ is defined by

$$
I_{4}(t):=\int_{0}^{t} \mathrm{e}^{(t-s) A} \sigma(s) \mathrm{d} Z(s)=\sum_{k=1}^{\infty}\left(\int_{0}^{t} \beta_{k} \mathrm{e}^{-\lambda_{k}(t-s)} \sigma(s) \mathrm{d} W^{k}(S(s))\right) e_{k} .
$$

By the Gaussian formula, following an argument like that of [10, Proposition 4.2] and (2.11), we get

$$
\left(\mathbb{E}\left(\left\|I_{4}(t)\right\|_{\mathbb{H}}^{p}\right)\right)^{1 / p} \leq C\left(\sum_{k=1}^{\infty} \int_{0}^{t} \beta_{k}^{2} \mathrm{e}^{-2 \lambda_{k}(t-s)} \sigma^{2}(s) \mathrm{d} s\right)^{1 / 2} t^{\frac{1}{\alpha}-\frac{1}{2}},
$$


where $C>0$ is some constant. In view of (2.8), (2.9), and (3.5), it follows from (3.4) that

$$
\begin{aligned}
\mathrm{e}^{\lambda_{1} t}\left(\mathbb{E}\left(\|X(t)\|_{\mathbb{H}}^{p}\right)\right)^{1 / p} \leq & \|x\|_{\mathbb{H}}+L_{2} \int_{0}^{t} \mathrm{e}^{\lambda_{1} s}\left\{\gamma(s)+\left(\mathbb{E}\left(\|X(s)\|_{\mathbb{H}}^{p}\right)\right)^{1 / p}\right\} \mathrm{d} s \\
& +C^{1 / p} \mathrm{e}^{\lambda_{1} t}\left(\sum_{k=1}^{\infty} \int_{0}^{t} \beta_{k}^{2} \mathrm{e}^{-2 \lambda_{k}(t-s)} \sigma^{2}(s) \mathrm{d} s\right)^{1 / 2} t^{\frac{1}{\alpha}-\frac{1}{2}} .
\end{aligned}
$$

Then, for arbitrary $\varepsilon>0$ sufficiently small, one has

$$
\begin{aligned}
\mathrm{e}^{\left(\lambda_{1}-\varepsilon\right) t}\left(\mathbb{E}\left(\|X(t)\|_{\mathbb{H}}^{p}\right)\right)^{1 / p} \leq & \|x\|_{\mathbb{H}}+L_{2} \int_{0}^{t} \mathrm{e}^{\lambda_{1} s} \gamma(s) \mathrm{d} s+L_{2} \int_{0}^{t} \mathrm{e}^{\left(\lambda_{1}-\varepsilon\right) s}\left(\mathbb{E}\left(\|X(s)\|_{\mathbb{H}}\right)^{p}\right)^{1 / p} \mathrm{~d} s \\
& +C^{1 / p} \mathrm{e}^{\lambda_{1} t}\left(\sum_{k=1}^{\infty} \int_{0}^{t} \beta_{k}^{2} \mathrm{e}^{-2 \lambda_{k}(t-s)} \sigma^{2}(s) \mathrm{d} s\right)^{1 / 2} t^{\frac{1}{\alpha}-\frac{1}{2}} \mathrm{e}^{-\varepsilon t}
\end{aligned}
$$

Thanks to (2.4), (3.3), and the uniform boundedness of $t^{\frac{1}{\alpha}-\frac{1}{2}} \mathrm{e}^{-\varepsilon t}$ by $\alpha \in(1,2)$ and $\varepsilon>0$, there exists $\bar{C}>0$ such that

$$
\mathrm{e}^{\left(\lambda_{1}-\varepsilon\right) t}\left(\mathbb{E}\left(\|X(t)\|_{\mathbb{H}}^{p}\right)\right)^{1 / p} \leq \bar{C}+L_{2} \int_{0}^{t} \mathrm{e}^{\left(\lambda_{1}-\varepsilon\right) s}\left(\mathbb{E}\left(\|X(s)\|_{\mathbb{H}}\right)^{p}\right)^{1 / p} \mathrm{~d} s
$$

This, together with the Gronwall inequality, leads to

$$
\mathrm{e}^{\left(\lambda_{1}-\varepsilon\right) t}\left(\mathbb{E}\left(\|X(t)\|_{\mathbb{H}}^{p}\right)\right)^{1 / p} \leq \bar{C} \mathrm{e}^{L_{2} t}
$$

Then, by $L_{2} \in\left(0, \lambda_{1}\right)$, we take $\varepsilon>0$ such that $\lambda_{1}-L_{2}-\varepsilon>0$ and the desired assertion follows immediately.

Remark 3.1 We remark that Example 2.3 still satisfies the condition (3.3).

In what follows, we further apply the technique adopted in the argument of Theorem 3.1 to study the stability for a class of SPDEs driven by fractional Brownian motion. We also need to recall some details of fractional Brownian motion. Let $B^{H}(t)$ be an $\mathbb{H}$-valued cylindrical fractional Brownian motion defined by

$$
B^{H}(t):=\sum_{k=1}^{\infty} \beta_{k}^{H}(t) e_{k}, \quad t \geq 0
$$

Here, $\left\{\beta_{k}^{H}\right\}_{k \geq 1}, H \in(0,1)$, are $\mathbb{R}$-valued independent fractional Brownian motions with Hurst index $H$, i.e., for each $k>0, \beta_{k}^{H}$ is an $\mathbb{R}$-valued centered Gaussian process starting from zero, defined on the probability space $\left(\Omega, \mathcal{F},\left\{\mathcal{F}_{t}\right\}_{t \geq 0}, \mathbb{P}\right)$, with covariance function

$$
R(t, s):=\mathbb{E}\left[\beta_{k}^{H}(t) \beta_{k}^{H}(s)\right]=\left(t^{2 H}+s^{2 H}-|t-s|^{2 H}\right) / 2, \quad t, s \geq 0,
$$

and $\left\{e_{k}\right\}_{k \geq 1}$ is an orthonormal basis of $H .\left\{\beta_{k}^{H}\right\}_{k \geq 1}$ are standard Brownian motions for $H=\frac{1}{2}$, admit self-similarity, possess Hölder continuity, but are not semi-martingales for 
$H \neq \frac{1}{2}$; see, e.g., [16, Chapter 5]. To the best of our knowledge, there are essentially two different approaches to construct stochastic integrals with respect to the fractional Brownian motion, i.e., a path-wise approach and the Malliavin calculus; see, e.g., the monograph [16].

For any $T>0, H>\frac{1}{2}$ and $\sigma:[0, \infty) \rightarrow L(\mathbb{H}, \mathbb{H})$, the family of linear operators from $\mathbb{H}$ to $\mathbb{H}$, the stochastic integral with respect to the cylindrical fractional Brownian motion is defined by

$$
\int_{0}^{t} \sigma(s) \mathrm{d} B^{H}(s):=\sum_{k=1}^{\infty} \int_{0}^{t} \sigma(s) e_{k} \mathrm{~d} \beta_{k}^{H}(s)=\sum_{k=1}^{\infty} \int_{0}^{t}\left(K_{H}^{*}\left(\sigma e_{k}\right)\right)(s) \mathrm{d} W_{k}(s), \quad t \in[0, T]
$$

provided that $\sum_{k=1}^{\infty} \int_{0}^{T}\left\|K_{H}^{*}\left(\sigma e_{k}\right)(t)\right\|_{\mathbb{H}}^{2} \mathrm{~d} t<\infty$. Here, for each $k \geq 1, \beta_{k}^{H}$ admits the Wiener integral representation:

$$
\beta_{k}^{H}(t)=\int_{0}^{t} K^{H}(t, s) \mathrm{d} W_{k}(s)
$$

where $W_{k}$ is an $\mathbb{R}$-valued Wiener process, and the kernel $K^{H}$ is given by

$$
K^{H}(t, s):=c_{H} s^{1 / 2-H} \int_{s}^{t}(u-s)^{H-3 / 2} u^{H-1 / 2} \mathrm{~d} u, \quad s<t
$$

for some positive constant $c_{H}$, and, for $\phi:[0, T] \rightarrow \mathbb{H}$,

$$
\left(K_{H}^{*} \phi\right)(t):=\int_{t}^{T} \phi(s) \frac{\partial K_{H}}{\partial s}(s, t) \mathrm{d} s, \quad t \in[0, T] .
$$

For more details of the stochastic integration with respect to the infinite-dimensional fractional Brownian motion, we refer to, e.g., Tindel et al. [17].

In the following section, we consider an SPDE driven by the cylindrical fractional Brownian motion $B^{H}(t)$, defined by (3.6), on $\mathbb{H}$

$$
\mathrm{d} X(t)=\{A X(t)+b(t, X(t))\} \mathrm{d} t+\sigma(t) \mathrm{d} B^{H}(t), \quad t>0
$$

with initial value $X(0)=x$, where $A$ and $b$ are defined as in $(2.2)$ and $\sigma:[0,8) \rightarrow L_{H S}(\mathbb{H}, \mathbb{H})$, the space of all Hilbert-Schmidt operators, such that

$$
\int_{0}^{T}\|\sigma(t)\|_{H S}^{2} \mathrm{~d} t<\infty \quad \text { for any } T>0
$$

where $\|\cdot\|_{H S}$ denotes the usual Hilbert-Schmidt norm, i.e., $\|T\|_{H S}:=\left(\sum_{i=1}^{\infty}\left\|T e_{i}\right\|_{\mathbb{H}}^{2}\right)^{1 / 2}$ for a Hilbert-Schmidt operator. Under (A1), (A2), and (3.8), (3.7) has a unique mild solution; see, e.g., [18, Theorem 1], that is, there exists a predictable $H$-valued stochastic process $X(t)$ such that

$$
X(t)=\mathrm{e}^{t A} x+\int_{0}^{t} \mathrm{e}^{(t-s) A} b(X(s)) \mathrm{d} s+\int_{0}^{t} \mathrm{e}^{(t-s) A} \sigma(s) \mathrm{d} B^{H}(s) .
$$

Note that the solution of (3.7) is even not a semi-martingale so that the Itô formula is unavailable.

The other main results in this section is as follows. 
Theorem 3.2 Let (A1), (A2), and (3.8) hold and assume further that

$$
\tilde{\delta}:=\sup _{t \geq 0}\left(\int_{0}^{t} \mathrm{e}^{2 s \lambda_{1}}\|\sigma(s)\|_{H S}^{2} \mathrm{~d} s\right)<\infty
$$

Then, for $H>\frac{1}{2}$ and $L \in\left(0, \lambda_{1}\right)$, there exist $c_{3}>0$ and $\gamma \in\left(0, \lambda_{1}-L\right)$ such that

$$
\mathbb{E}\left(\|X(t)\|_{\mathbb{H}}^{2}\right) \leq c_{3} \mathrm{e}^{-2 \gamma t}
$$

In other words, the solution is exponentially stable in mean square, where the Lyapunov exponent is $-\gamma$.

Proof We here only outline the argument of Theorem 3.2 since it is similar to that of Theorem 3.1. Recall from [18, Lemma 2] that

$$
\begin{aligned}
\mathbb{E}\left\|\int_{0}^{t} \mathrm{e}^{(t-s) A} \sigma(s) \mathrm{d} B^{H}(s)\right\|_{\mathbb{H}}^{2} & \leq c H(2 H-1) t^{2 H-1} \int_{0}^{t}\left\|\mathrm{e}^{(t-s) A} \sigma(s)\right\|_{H S}^{2} \mathrm{~d} s \\
& \leq c H(2 H-1) t^{2 H-1} \int_{0}^{t} \mathrm{e}^{-2(t-s) \lambda_{1}}\|\sigma(s)\|_{H S}^{2} \mathrm{~d} s
\end{aligned}
$$

for some constant $c>0$, due to the fact that $\mathrm{e}^{t A}$ is a bounded linear operator and $\sigma$ is Hilbert-Schmidt. Following the argument of Theorem 3.1, for any $\varepsilon>0$ sufficiently small, we have

$$
\begin{aligned}
\mathrm{e}^{\left(\lambda_{1}-\varepsilon\right) t}\left(\mathbb{E}\left(\|X(t)\|_{\mathbb{H}}^{2}\right)\right)^{1 / 2} \leq & \|x\|_{\mathbb{H}}+L_{2} \int_{0}^{t} \mathrm{e}^{\lambda_{1} s} \gamma(s) \mathrm{d} s+L_{2} \int_{0}^{t} \mathrm{e}^{\left(\lambda_{1}-\varepsilon\right) s}\left(\mathbb{E}\left(\|X(s)\|_{\mathbb{H}}\right)^{2}\right)^{1 / 2} \mathrm{~d} s \\
& +\sqrt{c H(2 H-1)} t^{(2 H-1) / 2} \mathrm{e}^{-\varepsilon t}\left(\int_{0}^{t} \mathrm{e}^{2 s \lambda_{1}}\|\sigma(s)\|_{H S}^{2} \mathrm{~d} s\right)^{1 / 2} .
\end{aligned}
$$

Taking (2.4), (3.9), and the uniform boundedness of $t^{(2 H-1) / 2} \mathrm{e}^{-\varepsilon t}$ into account and thus applying the Gronwall inequality yields the desired assertion.

Remark 3.2 By the approach introduced in the previous section, we discuss the mean square exponential stability of (3.7) under the condition $L \in\left(0, \lambda_{1}\right)$. However, Caraballo et al. [18] investigated the same problem under $L_{2} \in\left(0,6^{-1 / 2} \lambda_{1}\right)$. In other words, we have improved some existing results in certain sense.

Remark 3.3 All the results in this paper can be further extended to functional SPDEs driven by $\alpha$-stable noise and fractional Brownian motion, including variable delay and distributed delay, while we here omit such discussions since there are no technical problems. Furthermore, there are also some interesting problems for SPDEs driven by $\alpha$-stable noise to be investigated, e.g., since (2.2) is non-autonomous, the mild solution is not a homogeneous Markov process, which makes the investigation of the stability in distribution of analytic solution, a weaker stability notion than exponential stability, and the corresponding numerical stability, very interesting. Such a topic will be reported in our forthcoming paper. 


\section{Competing interests}

The authors declare that they have no competing interests.

\section{Authors' contributions}

The main idea of this paper was proposed by JYW and YLR. JYW prepared the manuscript initially and performed all the steps of the proofs in this research. All authors read and approved the final manuscript.

\section{Acknowledgements}

This research is supported by the Natural Science Foundation of China under Grant no. 71372063.

\section{Received: 24 December 2013 Accepted: 18 March 2014 Published: 28 Mar 2014}

\section{References}

1. Bao, J, Yuan, C: Stabilization of partial differential equations by Levy noise. Stoch. Anal. Appl. 30, 354-374 (2012)

2. Bao, J, Mao, X, Yuan, C: Lyapunov exponents of hybrid stochastic heat equations. Syst. Control Lett. 61, 165-172 (2012)

3. Bao, J, Truman, A, Yuan, C: Almost sure asymptotic stability of stochastic partial differential equations with jumps. SIAM J. Control Optim. 49, 771-787 (2011)

4. Chow, P: Stochastic Partial Differential Equations. Chapman \& Hall/CRC, Boca Raton (2007)

5. Liu, K: Stability of Infinite Dimensional Stochastic Differential Equations with Applications. Chapman \& Hall/CRC, Boca Raton (2006)

6. Yuan, C, Bao, J: On the exponential stability of switching-diffusion processes with jumps. Q. Appl. Math. 71, 311-329 (2013)

7. Solomon, TH, Weeks, ER, Swinney, HL: Observation of anomalous diffusion and Lévy flights in a two-dimensional rotating flow. Phys. Rev. Lett. 71, 3975-3978 (1993)

8. Priola, E, Zabczyk, J: Structural properties of semilinear SPDEs driven by cylindrical stable processes. Probab. Theory Relat. Fields 149, 97-137 (2011)

9. Dong, Z, Xu, L, Zhang, X: Ergodicity of stochastic Burgers equations driven by $\alpha$-stable processes. arXiv:1208.5804v1

10. Zhang, X: Derivative formula and gradient estimation for SDEs driven by $\alpha$-stable processes. arXiv:1204.2630v2

11. Wang, F-Y: Gradient estimate for Ornstein-Uhlenbeck jump processes. Stoch. Process. Appl. 121, 466-478 (2011)

12. Wang, J: Harnack inequalities for Ornstein-Uhlenbeck processes driven by Lévy processes. arXiv:1105.2958v1

13. Applebaum, D: Lévy Processes and Stochastic Calculus, 2nd edn. Cambridge University Press, Cambridge (2009)

14. Sato, K: Lévy Processes and Infinite Divisible Distributions. Cambridge University Press, Cambridge (1999)

15. Kato, T: Perturbation Theory for Linear Operators. Springer, New York (1966)

16. Nualart, D: The Malliavin Calculus and Related Topics, 2nd edn. Springer, Berlin (2006)

17. Tindel, S, Tudor, C, Viens, F: Stochastic evolution equations with fractional Brownian motion. Probab. Theory Relat. Fields 127, 186-204 (2003)

18. Caraballo, T, Garrido-Atienza, MJ, Taniguchi, T: The existence and exponential behavior of solutions to stochastic delay evolution equations with a fractional Brownian motion. Nonlinear Anal. 74, 3671-3684 (2011) 